\title{
Fast and Slow Ligand Exchange at the Surface of Colloidal Gold Nanoparticles
}

\author{
Rebecca Dinkel ${ }^{1}$, Björn Braunschweig ${ }^{1,2} *$ and Wolfgang Peukert ${ }^{1,2}$ \\ ${ }^{1}$ Institute of Particle Technology (LFG), Friedrich-Alexander University Erlangen-Nürnberg \\ (FAU), Cauerstraße 4, 91058 Erlangen, Germany. \\ ${ }^{2}$ Cluster of Excellence - Engineering of Advanced Material (EAM), Friedrich-Alexander \\ University Erlangen-Nürnberg (FAU), Nägelsbachstraße 49b, 91058 Erlangen, Germany
}

\section{Experimental setups for SHS spectroscopy}

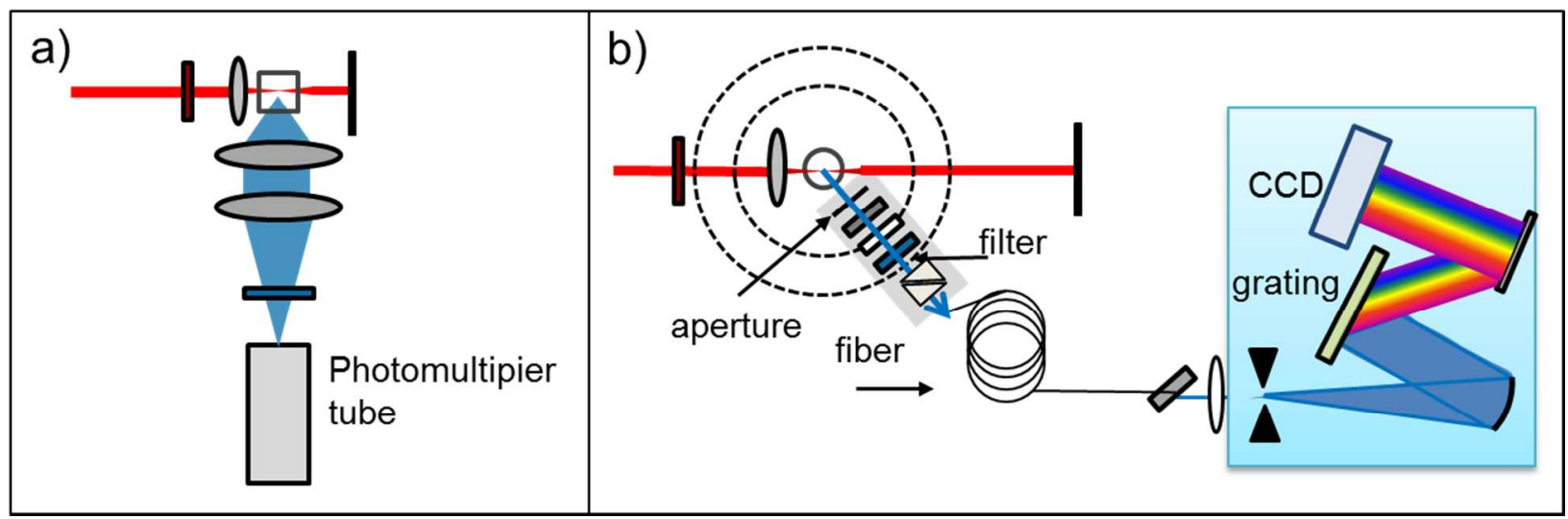

Figure S1 Schematic representation of the setups for SHS spectroscopy: a) High-throughput setup for in situ experiments which utilizes a photomultiplier tube for signal collecting; b) Setup for wavelength-resolved spectra which is based on fiber-optics and uses a spectrograph with a CCD camera for signal detection. Both setups use an excitation wavelength of $810 \mathrm{~nm}, 70 \mathrm{fs}$ pulse duration and $10 \mathrm{~nm}$ bandwidth. Signal detection occurred at a scattering angle of $90^{\circ}$. For details see experimental section. 


\section{Characterization of the SHS process of AuNP}

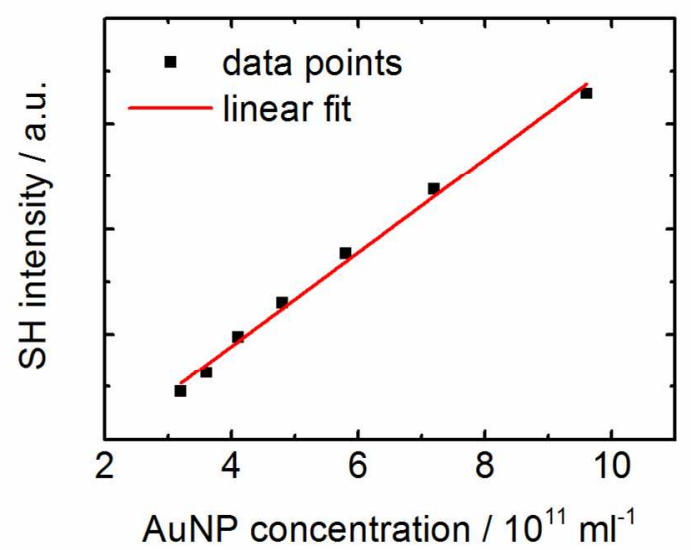

Figure S2 Concentration dependence of SHS intensity. The linear increase of the intensity with the AuNP concentration demonstrates that the scattering is a non-coherent process. The SHS intensity was corrected for 2-photon fluorescence and self-absorption. 

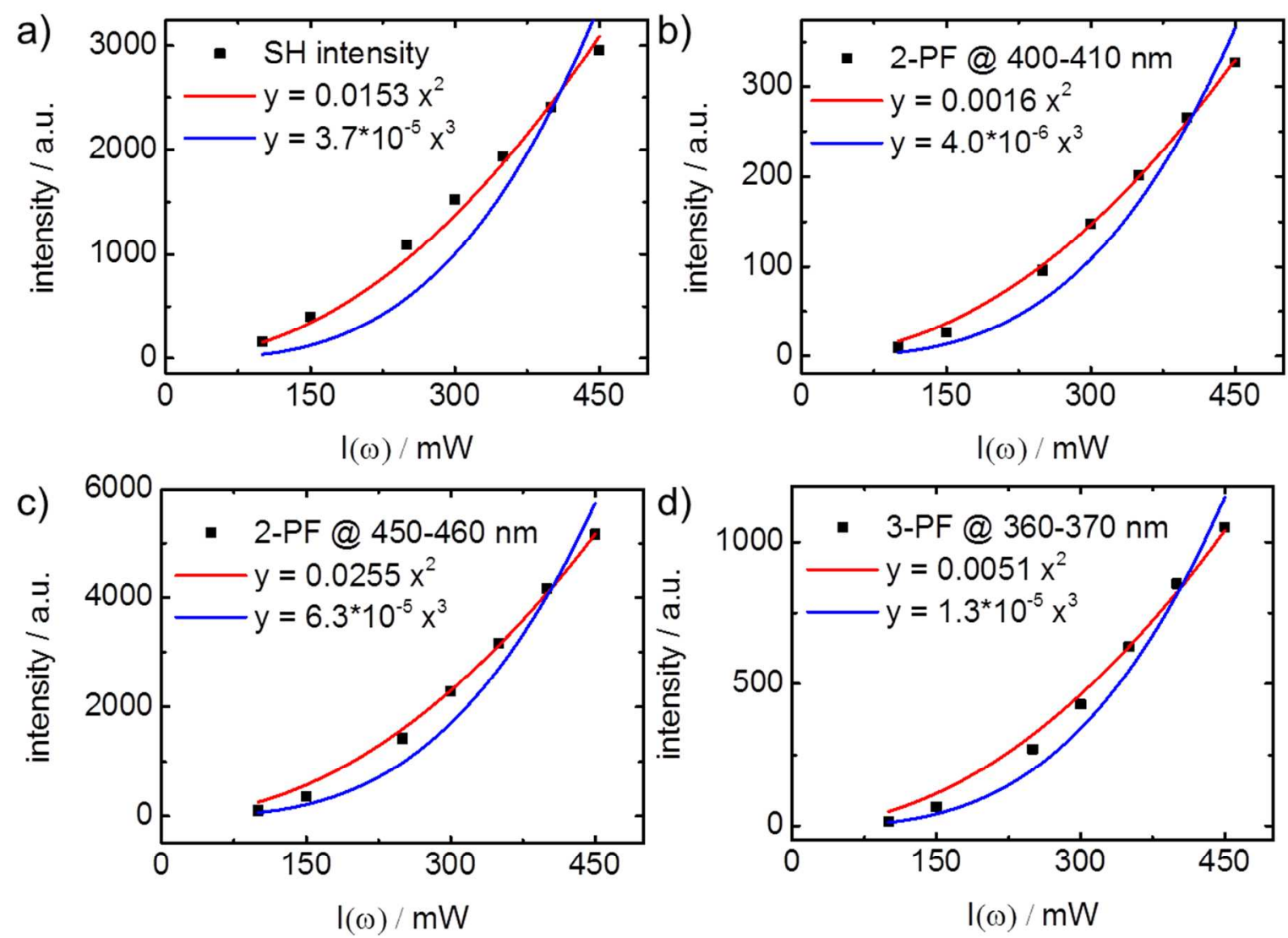

Figure S3 Power dependence of SHS (a) and fluorescence intensity of AuNPs at different wavelengths (b-d). Excitation wavelength was $810 \mathrm{~nm}$. Each data set has been fitted with a quadratic and a cubic function to determine the domination of a two- or three-photon process. The quadratic dependence proves that SHS at $400-410 \mathrm{~nm}$ (a) and the 2-PF at $400-410 \mathrm{~nm}$ (b) as well as at 450-460 nm (c) are two-photon processes. The fluorescence below $400 \mathrm{~nm}$ can be attributed to 3-photon fluorescence (3-PF) and higher order processes (d). 


\section{Comparison of SHS and 2-PF intensity during ligand exchange}

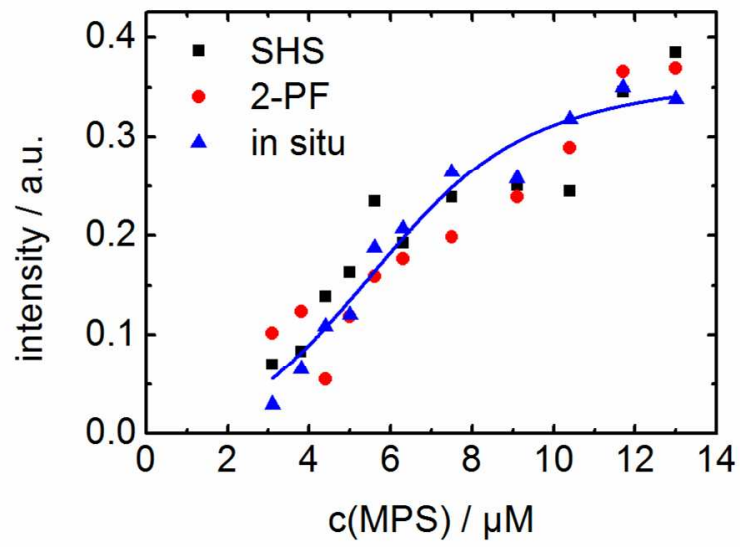

Figure S4 Comparison of the SHS and 2-PF intensity decrease of the wavelength resolved spectra and the intensity decrease of the in situ measurements at different MPS concentrations. It is found that the in situ results are in good agreement with the spectral results and also that the SHS intensity decrease is quite similar to the decrease of the 2-PF intensity. As discussed in the main text, the 2-PF is governed by two different mechanisms: charge-transfer of the organic molecule to the metal and direct donation of delocalized electrons. As two pathways are present the 2-PF is not linearly dependent on the MPS coverage. 


\section{Discussion of $\chi^{(3)}$ contributions to SHS intensity of AuNPs}

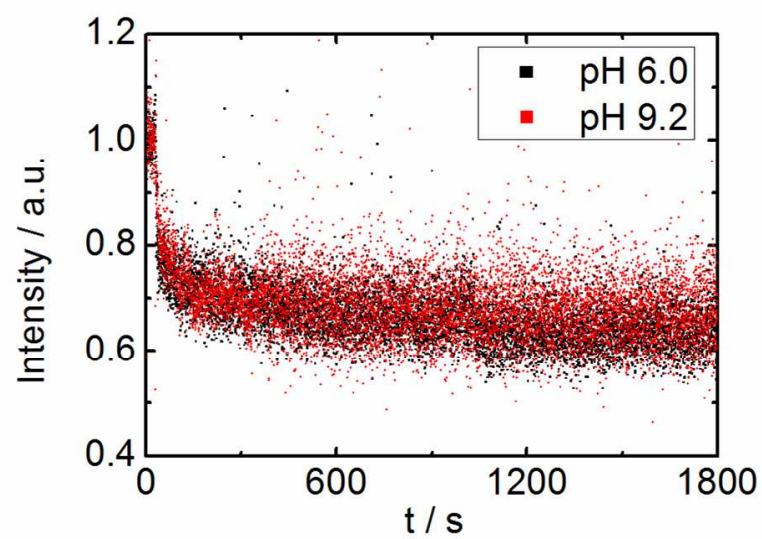

Figure S5 Ligand exchange on AuNPs with $17 \mu \mathrm{M}$ MPS at different pH. Ligand exchange was performed at a $\mathrm{pH}$ of 6.0 and 9.2 to determine whether different charging conditions at the $\mathrm{Au}$ surface had an effect on the overall SHS signals via $\chi^{(3)}$ contributions (Eq. (1)) and on exchange kinetics. The particle's zeta-potential decreased from $-59.8 \mathrm{mV}$ at $\mathrm{pH} 6.0$ to $-69.0 \mathrm{mV}$ for $\mathrm{pH} 9.2$ while the amount of adsorbed citrate is expected to remain constant between $\mathrm{pH} 6$ and 9.2. In fact, we find that the temporal decay of the intensity as well as the overall decrease is almost identical at both $\mathrm{pH}$ values. This shows that for the $27 \mathrm{~nm}$ Au nanoparticles in this work $\chi^{(3)}$ contributions can be neglected. 


\section{Details of the analysis of the adsorption kinetics}
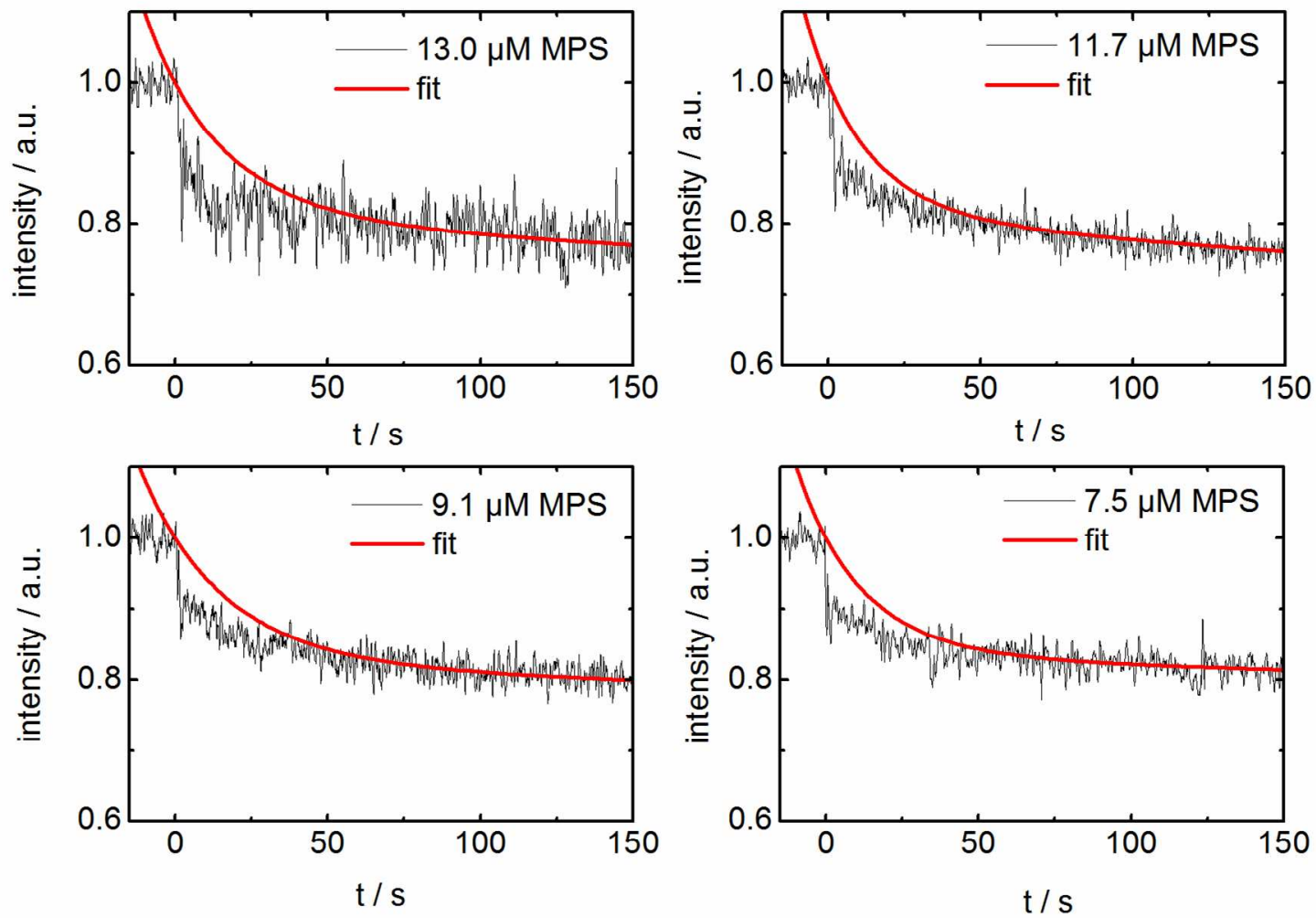

Figure S6 The kinetic data was fitted by the following equation:

(1) $\frac{I_{2 \omega}(t)}{I_{2 \omega}^{0}\left(t_{0}\right)}=\left(1-\left(A \cdot\left(1-e^{-k_{1} C t}\right)+B \cdot\left(1-e^{-k_{2} C t}\right)\right)\right)^{2}$

with $\mathrm{C}$ being the MPS concentration, $\mathrm{k}_{1}$ and $\mathrm{k}_{2}$ being the reaction constants for the fast and the slow process, A and B being the coverages for sites A and B. It is challenging to accurately fit the initial strong decrease; the best fit was obtained with the rate constants and parameters shown below. Changing either $\mathrm{k}_{1}$ or A led to a worse fit as indicated by an increased chi $^{2}$ (Figure S9). 


\begin{tabular}{|c|c|c|c|c|}
\hline MPS concentration $/ \mu \mathrm{M}$ & $\mathrm{k}_{1} / \mathrm{M}^{-1} \mathrm{~s}^{-1}$ & $\mathrm{k}_{2} / \mathrm{M}^{-1} \mathrm{~s}^{-1}$ & $\mathrm{~A}$ & $\mathrm{~B}$ \\
\hline 6.9 & 6254.5 & 208.8 & 0.078 & 0.129 \\
\hline 7.5 & 6081.0 & 167.8 & 0.067 & 0.086 \\
\hline 9.1 & 3918.1 & 249.8 & 0.050 & 0.092 \\
\hline 11.7 & 4288.2 & 210.8 & 0.097 & 0.097 \\
\hline 13.0 & 2983.2 & 148.6 & 0.095 & 0.099 \\
\hline
\end{tabular}

Table S1 Reaction constants and coverage parameters as obtained by the optimized fit shown above, for discussion see also below.

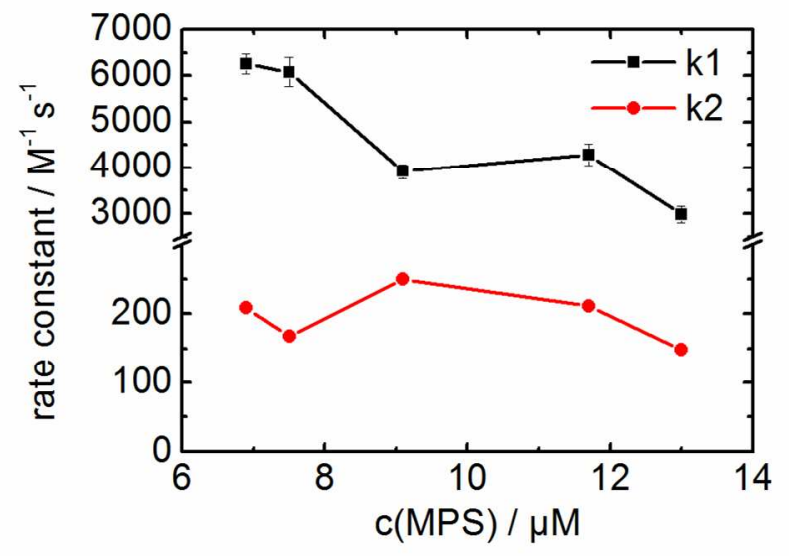

Figure S7 Rate constants $\mathrm{k}_{1}$ and $\mathrm{k}_{2}$ as obtained by the fit mentioned above versus MPS concentration. The rate constant $\mathrm{k}_{2}$ appears to be independent of the MPS concentration. The rate constant $\mathrm{k}_{1}$ scatters more strongly as the initial fast decrease cannot be properly represented by the fit. The rate constant $\mathrm{k}_{2}$ is attributed to the second step of the proposed adsorption model in which the MPS adsorbs to terrace sites while $\mathrm{k}_{1}$ is related to the adsorption of MPS to defect sites. 


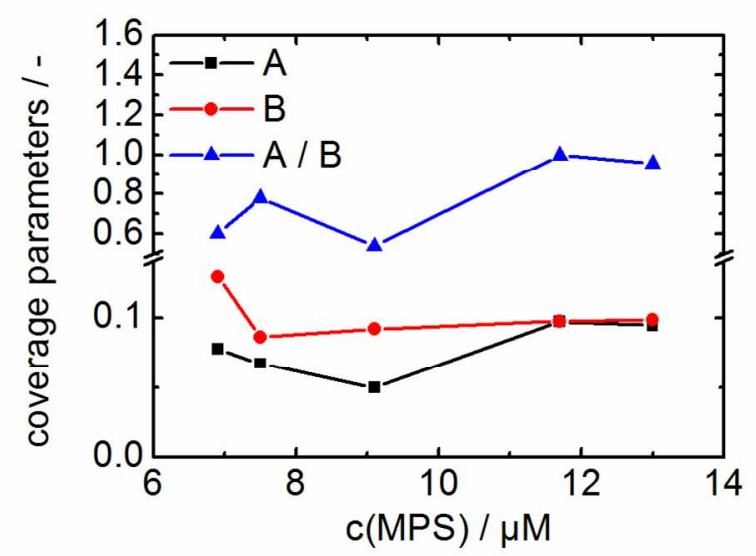

Figure S8 Coverage parameters A and B and their ratio A / B as obtained by the fit discussed above. For concentration below the saturation concentration of $\sim 11 \mu \mathrm{M}$ MPS the parameters as well as the ratio seem to scatter while for concentration of $11 \mu \mathrm{M}$ MPS and higher the constant appear to have reached a constant value. The ratio as these concentrations is about 1 which means that defect and terrace sites are equally filled by MPS.
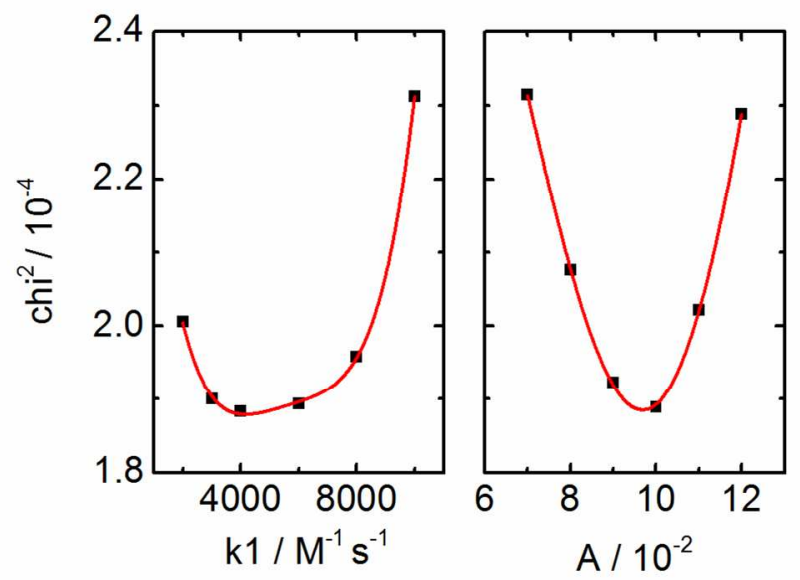

Figure S9 Deviation of $\mathrm{chi}^{2}$ upon variation of the fit parameters $\mathrm{k}_{1}$ and A. The fit parameters obtained at the minimized $\mathrm{chi}^{2}$ are presented in Table S1. 